\title{
Paediatric Crossword Puzzle 3 (Answers)
}

Manouri P. Senanayake ${ }^{1}$

Sri Lanka Journal of Child Health, 2009; 38: 44

\begin{tabular}{|c|c|c|c|c|c|c|c|c|c|c|c|c|}
\hline${ }^{1} \mathbf{A}$ & $\mathbf{M}$ & ${ }^{2} \mathbf{I}$ & $\mathbf{L}$ & O & ${ }^{3} R$ & I & D & ${ }^{4} \mathbf{E}$ & & $\mathbf{P}$ & & \\
\hline $\mathbf{L}$ & & $\mathbf{N}$ & & & $\mathbf{Y}$ & & & ${ }^{6} \mathbf{C}$ & $\mathbf{R}$ & $\mathbf{E}$ & $\mathbf{A}$ & $\mathbf{M}$ \\
\hline${ }^{8} \mathbf{P}$ & $\mathbf{E}$ & $\mathbf{N}$ & D & $\mathbf{R}$ & $\mathbf{E}$ & ${ }^{9} \mathbf{D}$ & & H & & $\mathbf{R}$ & & $\mathbf{A}$ \\
\hline $\mathbf{O}$ & & $\mathbf{O}$ & & & 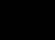 & ${ }^{10} \mathbf{I}$ & $\mathbf{L}$ & O & & $\mathbf{L}$ & & $\mathbf{L}$ \\
\hline${ }^{11} \mathbf{R}$ & $\mathbf{O}$ & $\mathbf{C}$ & $\mathbf{K}$ & I & $\mathbf{N}$ & $\mathbf{G}$ & & & & ${ }^{12} \mathbf{S}$ & B & $\mathbf{R}$ \\
\hline $\mathbf{T}$ & & $\mathbf{E}$ & & & & ${ }^{13} \mathbf{E}$ & V & ${ }^{14} \mathbf{A}$ & $\mathbf{N}$ & & & $\mathbf{O}$ \\
\hline & ${ }^{15} \mathbf{A}$ & $\mathbf{N}$ & $\mathbf{P}$ & & 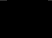 & $\mathbf{O}$ & & $\mathbf{M}$ & & ${ }^{6} \mathrm{C}$ & $\mathbf{A}$ & $\mathbf{T}$ \\
\hline${ }^{7} \mathbf{S}$ & $\mathbf{A}$ & $\mathbf{T}$ & & ${ }^{18} \mathbf{H}$ & $\mathbf{E}$ & $\mathbf{R}$ & $\mathbf{A}$ & $\mathbf{L}$ & D & & & $\mathbf{A}$ \\
\hline $\mathbf{C}$ & & & & $\mathbf{E}$ & & $\mathbf{G}$ & & & 0 & & ${ }^{20} \mathbf{I}$ & $\mathbf{T}$ \\
\hline${ }^{21} \mathbf{U}$ & $\mathbf{K}$ & & B & $\mathbf{L}$ & $\mathbf{E}$ & $\mathbf{E}$ & ${ }^{23} \mathbf{D}$ & I & $\mathbf{N}$ & $\mathbf{G}$ & & I \\
\hline $\mathbf{R}$ & & ${ }^{24} \mathbf{A}$ & & $\mathbf{P}$ & & & $\mathbf{M}$ & & 0 & & & O \\
\hline${ }^{25} \mathrm{~V}$ & $\mathbf{A}$ & $\mathbf{C}$ & $\mathbf{T}$ & $\mathbf{E}$ & $\mathbf{R}$ & $\mathbf{L}$ & & & ${ }^{26} \mathbf{R}$ & $\mathbf{A}$ & ${ }^{27} \mathbf{I}$ & $\mathbf{N}$ \\
\hline $\mathbf{Y}$ & & $\mathbf{E}$ & & ${ }^{28} \mathbf{R}$ & $\mathbf{A}$ & B & I & $\mathbf{E}$ & $\mathbf{S}$ & & $\mathbf{M}$ & \\
\hline
\end{tabular}

${ }^{1}$ Professor of Paediatrics, Faculty of Medicine, University of Colombo 